\section{A case report of Neisseria mucosa peritonitis in a chronic ambulatory peritoneal dialysis patient}

\author{
Alan Awdisho, Maria Bermudez \\ Geisinger Medical Center, Danville, PA, \\ USA
}

\begin{abstract}
Peritonitis is a leading complication of chronic ambulatory peritoneal dialysis. However, very rarely does Neisseria mucosa cause peritonitis. We describe an unusual case of $N$. mucosa peritonitis in a chronic ambulatory peritoneal dialysis patient. A 28-year-old Hispanic male presents with diffuse abdominal pain exacerbated during draining of the peritoneal fluid. Peritoneal fluid examination was remarkable for leukocytosis and gramnegative diplococci. Bacterial cultures were positive for $N$. mucosa growth. The patient was treated with ciprofloxacin with preservation of the dialysis catheter. This case highlights the rarity and importance of Neisseria mucosa causing peritonitis in chronic ambulatory peritoneal dialysis patients'. There seems to be a unique association between $N$. mucosa peritonitis and chronic ambulatory peritoneal dialysis patients'. The patient was successfully managed with ciprofloxacin along with salvaging of the dialysis catheter.
\end{abstract}

\section{Introduction}

Traditionally, the Neisseria species has two potential pathogenic strains; Neisseria meningitidis and Neisseria gonorrhoeae. The remainder of Neisseria species, including Neisseria mucosa, are considered normal flora colonizing the respiratory tract, living in symbiosis. ${ }^{1}$ One analysis showed that the majority of people are colonized by one to two of these Neisseria species. ${ }^{2}$ However, this traditional dogma considering these Neisseria species nonpathogenic is recently being questioned. Specifically, Neisseria mucosa is increasingly found to cause a multitude of diseases, in particular peritonitis.

Part of the difficulty in associating disease with other species of Neisseria, such as Neisseria mucosa, is the fact that they do not contain the typical virulence factors. The established pathogens Neisseria meningitidis and Neisseria gonorrhoeae derive much of their virulence from attaching to mucosal surfaces. ${ }^{3}$ However, the lack of these virulence factors does not necessarily make the other Neisseria species benign.

The advent of Chronic Ambulatory Peritoneal Dialysis (CAPD) has permitted significant patient autonomy with the concomitant risk of bacterial peritonitis. The typical culprits are gram-positive species, such as Staphylococcus species and Streptococcus species. Gram-negative organisms account for up to $25 \%$ of cases. ${ }^{4}$

We performed a PubMed literature search using the terms Neisseria mucosa, Peritonitis, and Chronic Ambulatory Peritoneal Dialysis. This yielded very few published cases of CAPD patients' afflicted by Neisseria mucosa peritonitis. $^{5-8}$ In this clinical vignette, we add another case of CAPD associated with Neisseria mucosa peritonitis.

\section{Case Report}

The patient was a 28 year-old Hispanic man with a history of end stage renal disease due to hypertensive disease since the age of 10 years, complicated by malignant hypertension. His surgical history was remarkable for a remotely failed renal transplant requiring an allograft nephrectomy. He had been on hemodialysis for about a decade since, and transitioned to peritoneal dialysis just six months prior to presenting to our facility with sepsis due to new-onset peritonitis.

In the outpatient clinic, the patient had been diagnosed with external cheek cellulitis that responded to a short course of empiric cephalexin. He overcame this only to experience three days of abdominal pain near his dialysis catheter. The pain was constant and severe pain, worsened by draining and filling during dialysis sessions. He denied any fevers, chills, nausea, vomiting, diarrhea, oropharyngeal and genitourinary complaints or decrease in recent ultrafiltration. Exam was remarkable for a blood pressure of $167 / 99 \mathrm{mmHg}$, heart rate of 107 beats per minute and abdominal tenderness to palpation diffusely with the catheter exit site clear dry and intact without exudate or erythema.

He quickly underwent peritoneal fluid examination, revealing an absolute neutrophil count of 14,458 cells $/ \mathrm{mm}^{3}$, confirming the diagnosis of bacterial peritonitis. His gram stain revealed gram-negative diplococci and subsequent chocolate agar plated cultures grew Neisseria species, not gonorrhoeae or meningitidis. Further biochemical speciation (via RapID NH System by Remel, Thermo Fisher Scientific Inc., Waltham, USA) showed Neisseria mucosa to be the etiology of the patient's bacterial peritonitis. Given this is typically nonpathogenic, further susceptibility was not performed.

The patient was placed on empiric intraperi-
Correspondence: Alan Awdisho, Abrazo West Valley Campus, 13677 W McDowell Rd, Goodyear, AZ, 85395, USA.

Tel.: +1.623.882.1500 - Fax: +1.623 .882 .1683 .

E-mail: aawdisho@soundphysicians.com

Key words: Neisseria mucosa; Peritonitis; Chronic Ambulatory Peritoneal Dialysis.

Acknowledgments: the authors would thank Mary Kay, Library Specialist.

Contributions: AA contributed to the concept and design and acquiring and analyzing the data of the study. MB contributed by interpreting the data and revising the draft. Both authors approved the final version.

Conflict of interest: the authors declare no potential conflict of interest.

Received for publication: 28 0ctober 2016.

Revision received: 2 November 2016.

Accepted for publication: 2 November 2016.

This work is licensed under a Creative Commons Attribution-NonCommercial 4.0 International License (CC BY-NC 4.0).

(O) Copyright A. Awdisho and M. Bermudez., 2016 Licensee PAGEPress, Italy

Infectious Disease Reports 2016; 8:6950

doi:10.4081/idr.2016.6950

toneal vancomycin 1 gram and cefepime 1 gram therapy and later transitioned to oral ciprofloxacin $500 \mathrm{mg}$ daily for two weeks. During the hospitalization, repeat peritoneal fluid analysis confirmed a resolving leukocytosis and bacterial cultures without growth. Subsequently, the intraperitoneal catheter was preserved without recurrence of peritonitis to date.

\section{Discussion and Conclusions}

The likely source of Neisseria mucosa contamination of the dialysis port was the oral cellulitis. The transmission from the oral site to catheter site is supported by the well-known colonization of the oropharyngeal tract. CAPD seems to be associated with an increased risk for Neisseria mucosa, particularly in cases of peritonitis.

In general, this case adds to the current literature highlighting the importance of considering all types of disease caused by traditionally-benign Neisseria mucosa species. Although, nongonococcal and nonmeningococcal Neisseria strains are usually benign and infections are rare, they may cause a variety of disease.

This case highlights the danger of Neisseria 
mucosa peritonitis in patients' undergoing CAPD, and is the fourth reported case. The rarity of case reports and lack of the established pathogenic factors of nongonococcal and nonmeningococcal Neisseria may limit recognition of these infections. ${ }^{9}$ This makes the association with clinical disease complex, and further investigation regarding their pathogenicity is warranted.

In previously described case reports of nongonococcal and nonmeningococcal Neisseria, patients were treated with ciprofloxacin. ${ }^{10}$ Our patient was also successfully treated with ciprofloxacin. Given the increasing frequency of infections by nongonococcal and nonmeningococcal Neisseria, guidelines regarding appropriate antimicrobial therapy should be established. Further case reports should also establish the safety of preserving the peritoneal dialysis catheters, as in our patient's case.

\section{References}

1. Ryan KJ, Ray C, edsNeisseria. In: Sherris medical microbiology. 6th Ed. New York: McGraw-Hill; 2014. 2. Sáez Nieto JA, Marcos C, Vindel A. Multicolonization of human nasopharynx due to Neisseria spp. Int Microbiol 1998;1:59-63.

3. Stephens DS, McGee ZA, Melly MA, et al. Attachment of pathogenic Neisseria to human mucosal surfaces: role in pathogenesis. Infection 1982;10:192-5.

4. Papadakis MA, McPhee SJ, Rabow MW. Current medical diagnosis and treatment. 2015. New York, NY: McGraw-Hill; 2014.

5. Shetty, AK, Nagaraj SK, Lorentz WB, Bitzan M. Peritonitis due to Neisseria mucosa in an adolescent receiving peritoneal dialysis. Infection 2005;33:390-2.

6. Macia M, Vega N, Elucaz R, et al. Neisseria mucosa peritonitis in CAPD: another case of the "nonpathogenic" Neisseriae infec- tion. Perit Dial Int 1993;13:72-3.

7. Konner P, Watschinger B, Apfalter P, et al. A case of continuous ambulatory peritoneal dialysis peritonitis with an uncommon organism and an atypical clinical course. Am J Kidney Dis 2001;37:E10.

8. Lee WC, Yang WC, Chen TW, et al. Unusual presentation of Neisseria mucosa peritonitis with persistent ultrafiltration failure and clear effluent. Perit Dial Int 2003;23:198-9.

9. Stotka JL, Rupp ME, Meier FA, Markowitz SM. Meningitis due to Neisseria mucosa: case report and review. Rev Infect Dis 1991;13:837-41.

10. Taegtmeyer M, Saxena R, Corkill JE, et al. Ciprofloxacin treatment of bacterial peritonitis associated with chronic ambulatory peritoneal dialysis caused by Neisseria cinerea. J Clin Microbiol 2006;44:3040-1. 